\title{
Pesquisa, Desenvolvimento e Avaliação de um Jogo para o Ensino de Matemática, Baseado no Processo de Virtualização de Jogos
}

\author{
Wilk Oliveira dos Santos $^{12}$, Clovis Gomes da Silva Junior ${ }^{1}$ \\ ${ }^{1}$ Universidade de Pernambuco, Campus Garanhuns (UPE) Av. Cap. Pedro \\ Rodrigues, 105 - São José, CEP 55.294-902 - Garanhuns - PE - Brasil \\ ${ }^{2}$ Núcleo de Excelência em Tecnologias Sociais (NEES) - Instituto de Computação - \\ Universidade Federal de Alagoas (IC-UFAL) Av. Lourival Melo Mota, S/N - Tabuleiro \\ dos Martins, CEP 57072-900 - Maceió - AL - Brasil \\ wos@ic.ufal.br, clovis.gomes@upe.br
}

Resumo: Este artigo aborda um trabalho de conclusão de curso, realizado por uma equipe multidisciplinar formada por estudantes de graduação, mestrado e doutorado da Universidade de Pernambuco Campus Garanhuns, além de profissionais das áreas de Matemática, Pedagogia e Psicologia. Apresenta-se um jogo desenvolvido por meio do processo de Virtualização de Jogos, destacando seu processo de pesquisa, desenvolvimento, aplicação e avaliação no cerne dos aspectos computacionais de interface, bem como de seus aspectos pedagógicos, em uma avaliação focada no cliente e no especialista. A pesquisa constatou que o jogo virtualizado foi avaliado de forma positiva diante dos aspectos verificados, bem como que o Processo de Virtualização se mostrou eficaz diante dos objetivos propostos.

Abstract: This paper promotes a work of course conclusion, accomplished for an multidisciplinary team, composed undergraduate, master's and Ph.D. students of University of Pernambuco Campus Garanhuns, as well, professionals of Mathematic, Pedagogy and Psychology. We present a game developed by Game Virtualization Process, highlighting research process, development process, application and evaluation in interface and pedagogical aspects, the evaluation is focused in the client and specialist. The research found of the virtualized game, has evaluated positively by students and teachers, as well, the virtualization process is effective before the proposed objectives.

\section{Introdução}

A sociedade contemporânea é caracterizada pelo uso cada vez mais frequente das tecnologias digitais da informação e comunicação (TDIC) na organização das mais diferentes práticas sociais, como aponta Ribeiro (2012). Nesse contexto, o investimento em tecnologias e a presença das mesmas em salas de aula é cada vez maior. Comumente, encontram-se nas escolas (laboratórios de informática) ou até mesmo com os próprios estudantes, dispositivos como notebooks, tablets, smartphones, entre outros.

A integração destas tecnologias no ambiente escolar, possibilita discussões quanto aos métodos e práticas sobre o uso destas ferramentas para auxiliar o processo de ensino e aprendizagem, como aponta Silva Neto et al. (2013), suscitando a busca por alternativas, para, através do uso destas tecnologias, obter maneiras de facilitar os processos de ensino e aprendizagem. 


\section{CBIE-LACLO 2015}

Anais dos Workshops do IV Congresso Brasileiro de Informática na Educação (CBIE 2015)

Entre os métodos utilizados com esta finalidade, encontra-se a utilização de jogos tradicionais ${ }^{1}$ e/ou jogos digitais. Estes, de acordo com Sá et al. (2007) quando utilizados nas atividades de ensino, podem propiciar momentos lúdicos e interativos no processo de ensino e aprendizagem, tornando-se uma possibilidade de atrair a atenção dos estudantes. Buchinger et al. (2012) apontam que este tipo de mídia atrai com facilidade este público, já Reinoso et al. (2012) ratificam que os jogos digitais tornam a aprendizagem interessante e divertida.

Atualmente, pesquisas demonstram uma série de benefícios ao incluir os jogos digitais no processo ensino-aprendizagem, apontando possibilidades de ser um rico instrumento para a construção do conhecimento, transformando o ato de jogar em ato de aprender e ensinar, construindo os objetivos necessários para se alcançar a aprendizagem, como elucidado por Medeiros et al. (2013).

Contudo, observam-se nas discussões sobre o uso de jogos na educação, que podem existir também fatores negativos. Para Falkembach (2007) apud Medeiros et al. (2013), mesmo um jogo bem projetado pode ter algumas desvantagens como: se não for bem aplicado perde o objetivo; nem todos os conceitos podem ser explicados por meio dos jogos; se o professor interferir com frequência, perde a ludicidade; e se o aluno for obrigado a jogar por exigência do professor, o aluno fica contrariado.

As experiências de Cabreira e Aquino (2006) tratam do processo de virtualização do saber em uma perspectiva de substituição das atividades tradicionais por atividades ligadas as tecnologias digitais, apontando inclusive, que os professores por vezes se sentem impotentes ao levar um jogo tradicional a sala de aula, e os estudantes não aderem à atividade, pois segundo Santos et al. (2013) estes estudantes se sentem mais atraídos por atividades ligadas às TDIC em detrimento dos meios tradicionais.

Com o objetivo de minimizar esta dificuldade, observam-se na literatura, trabalhos que tratam da Virtualização de Jogos para o ensino. De acordo com Santos et al. (2013), através desta técnica é possível unir os princípios pedagógicos contidos nos jogos tradicionais, outrora usados como suporte ao ensino, às novas mídias presentes no contexto social atual. Nesta pesquisa, verificou-se que o fracasso de muitos jogos digitais voltados à educação tem como causa o fato de não apresentarem aparatos pedagógicos e psicopedagógicos que eram observados nos jogos tradicionais.

Neste contexto, este artigo almeja apresentar o processo de pesquisa, virtualização, aplicação e avaliação do jogo Conquistando com o Resto como uma possibilidade para o ensino do conceito de divisão, o qual se apodera dos princípios pedagógicos e psicopedagógicos contidos em sua versão tradicional ao passo que contempla ainda elementos e mecânicas de jogos contemporâneos, e demonstra, mediante a avaliação com os usuários, que atende tanto aos requisitos desejados em relação aos aspectos computacionais quanto atinge seus objetivos educacionais.

\section{Trabalhos Relacionados}

Cabreira e Aquino (2006) tratam da "virtualização do saber" abordando a união necessária entre o tradicional e o digital, destacando em uma abordagem teórica a

\footnotetext{
${ }^{1}$ Alguns autores usam outros termos para definir o que chamamos de jogos tradicionais, como, jogos físicos, jogos concretos, jogos não digitais ou jogos de console.
} 


\section{CBIE-LACLO 2015}

Anais dos Workshops do IV Congresso Brasileiro de Informática na Educação (CBIE 2015)

lacuna existente entre o tradicional e o digital, apontando o processo de desuso dos jogos tradicionais em comparação aos jogos digitais.

Neste sentido, Araújo et al. (2011) abordam em seu trabalho, a virtualização do esporte, fazendo uma análise entre os processos de virtualização esportiva, ligados aos jogos eletrônicos, sua interferência na cultura corporal de movimento e quais as possíveis implicações para a Educação Física. Estes trabalhos tratam dos avanços tecnológicos em detrimento dos objetos tradicionais, abordando ainda os problemas causados no âmbito educacional pelo esquecimento do tradicional em função dos avanços tecnológicos, corroborando e reafirmando a realização deste trabalho.

Por sua vez, Togni et al. (2009) apresentam o processo de desenvolvimento de um jogo (apresentado como jogo presencial) utilizado em diversas práticas de ensino de Matemática em um conjunto de cidades do Rio Grande do Sul. Este jogo foi desenvolvido à luz da teoria da aprendizagem significativa, dentro da perspectiva de um grupo que analisa objetos de aprendizagem e suas implicações nos processos de ensino e aprendizagem em ciências exatas e busca disponibilizar estes objetos na região onde foi aplicado o trabalho. Os resultados são regionalizados e o jogo não está disponível para outros interessados.

O jogo tradicional, desenvolvido por pesquisadores da instituição, não apresenta nenhuma metodologia que justifique sua escolha e não são apresentados critérios rigorosos que justifiquem a metodologia de desenvolvimento utilizada, exceto no tocante à linguagem de programação utilizada. Também não são apresentadas avaliações ou validações do software em ambientes educacionais.

Os autores Zorzal et al. (2008), por sua vez, tratam da aprendizagem colaborativa em caráter presencial e remoto, dissertando a respeito das oportunidades geradas pelas inovações tecnológicas quanto a comunicação interpessoal, discutindo soluções para a interação em ambientes colaborativos presenciais e remotos, usando recursos para a comunicação interpessoal (chat, áudio e videoconferência) e Realidade Aumentada (RA). O seu trabalho é baseado na criação de uma versão digital para jogos tradicionais, utilizando recurso de Realidade Virtual e Aumentada. O trabalho, porém não apresenta metodologias específicas para a escolha dos jogos ou para o seu processo de desenvolvimento, focando apenas nos aspectos tecnológicos da Realidade Virtual e Aumentada.

Diferentemente, o Processo de Virtualização descrito neste trabalho apresenta uma metodologia que abrange desde o processo de escolha do jogo a ser virtualizado, o processo de desenvolvimento sob a ótica da virtualização de jogos, até a aplicação e avaliação do jogo em contextos escolares, evidenciando a cautela não apenas com os aspectos computacionais, mas, inclusive, com os aspectos pedagógicos.

\section{Metodologia}

O grupo de trabalho que apresenta este texto vem trabalhando nesta pesquisa ao longo dos anos de 2012 a 2015, (eg: Santos e Silva Junior (2013); Santos e Silva Junior (2014); Santos et al. (2013) Santos et al. (2014)). Nestes trabalhos, é percebida uma lacuna em relação aos aspectos pedagógicos e psicopedagógicos contidos nos jogos tradicionais, outrora utilizados como mecanismos de apoio ao ensino e aprendizagem da disciplina de Matemática e os jogos digitais, usados atualmente com este mesmo propósito. 
Neste sentido, a metodologia do trabalho ora apresentado, construiu-se da seguinte maneira: em um primeiro momento, buscaram-se trabalhos científicos em fontes acadêmicas que dissertassem sobre jogos tradicionais e/ou jogos digitais usados no ensino da Matemática, com intuito de obter-se resultados referentes ao estado da arte. A pesquisa realizada gerou uma comparação entre os aspectos pedagógicos e psicopedagógicos dos jogos tradicionais em relação aos jogos digitais, onde os resultados deste trabalho geraram um survey, servindo como base para a proposta de Virtualização de Jogos, ambos descritos em Santos e Silva Junior (2014).

Neste intuito, considerando os resultados desta pesquisa e análises de professores da disciplina de Matemática descritas por Santos et al. (2013) foram selecionados oito jogos tradicionais que não possuíam ainda versões digitais. Estes jogos foram analisados individualmente por uma equipe multidisciplinar composta por: dois professores de Matemática, um profissional de Psicologia e um de Pedagogia, além de profissionais de Computação, com o objetivo de estabelecer uma ordem de importância para a virtualização destes jogos, tomando por base suas experiências com o uso de jogos no ensino, bem como a literatura estudada (eg. Santos et al. (2013)). A partir destes resultados, o jogo escolhido para passar pelo processo de virtualização, foi o jogo "Conquistando com Resto".

$\mathrm{Na}$ sequencia do trabalho, ocorreu à avaliação do jogo, de acordo com Gladcheff et al (2011) a avaliação de um jogo educativo deve ser feita com metodologias específicas, pois a incorporação desses aplicativos só se justifica quando possibilita um avanço qualitativo nos processos de ensino-aprendizagem. Assim, a avaliação do jogo, seguiu uma abordagem especifica para avaliação (eg. Santos et al. (2015)). Neste sentido, a avaliação buscou verificar os aspectos computacionais de interface, bem como os aspectos pedagógicos do jogo, em uma avaliação focada no cliente e no especialista.

\section{O Jogo Conquistando com o Resto}

Conquistando com o Resto é um jogo de tabuleiro (Imagem 1) cujo objetivo pedagógico é apoiar a aprendizagem de divisão, propiciando aos estudantes uma forma lúdica de exercitar a divisão, lidando com todos os elementos presentes nesta operação aritmética.

O tabuleiro é formado por 48 casas numeradas não sequencialmente, as quais representam os dividendos. O jogador da vez deve lançar o dado e o resultado obtido representa o divisor. Efetuada a operação de divisão do número da casa pelo valor resultante do lançamento do dado, o resto da divisão representa a quantidade de casas que o jogador deve avançar. Diz-se vencedor àquele que alcançar a última posição do tabuleiro primeiro.

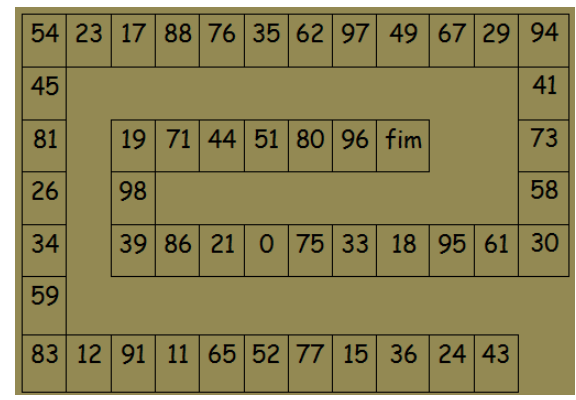

Imagem 1: Conquistando com Resto (versão tradicional) 


\section{Processo de Virtualização de Jogos}

O processo de Virtualização de Jogos é compreendido como o processo pelo qual os jogos tradicionais são recriados em versões digitais, de maneira que não percam seus aspectos pedagógicos e psicopedagógicos, os quais os levaram a serem utilizados como ferramenta de auxílio ao processo de ensino e aprendizado da disciplina em questão (Santos et al 2014).

Este processo envolve uma equipe multidisciplinar, abrangendo professores das disciplinas em questão, profissionais da área de Psicologia, além de profissionais de Pedagogia e Computação. Os profissionais de Psicologia são responsáveis por analisar e propor soluções para facilitar a interação dos estudantes e professores com os jogos, bem como manter os conceitos pedagógicos e psicopedagógicos contidos na versão tradicional do jogo. Os profissionais de Pedagogia e Computação, por sua vez, são responsáveis por atrelar os aspectos pedagógicos aos aspectos computacionais do jogo (Santos et al 2013).

\subsection{Processo de Virtualização do Jogo "Conquistando com Resto"}

Inicialmente, a primeira etapa do desenvolvimento (processo de virtualização) do jogo Conquistando com o Resto consistiu na análise do jogo por professores da disciplina de Matemática, considerando tanto o seu uso em sala de aula quanto as estruturas matemáticas abrangidas. Posteriormente, profissionais de psicologia definiram como seria a versão digital do jogo, contemplando uma mecânica que permitisse um uso agradável e satisfatório por parte de estudantes e professores, e que ao mesmo tempo, mantivesse os aspectos pedagógicos e psicopedagógicos da versão tradicional do jogo, fundamentais para que a versão digital do mesmo seja usada como recurso de apoio à aprendizagem da disciplina de Matemática.

Na sequência, ocorreu a escolha do software para desenvolvimento do jogo, a qual partiu da análise de quatro ferramentas de desenvolvimento de jogos digitais (eg. Santos et al. (2014)). A análise permitiu perceber as limitações de cada ferramenta, como, por exemplo, os tipos de gráficos produzidos, plataformas suportadas, dentre outros, conforme se pode observar no Quadro 1.

Quadro 1: Ferramentas Analisadas

\begin{tabular}{|l|l|l|}
\hline Ferramenta & Tipos de Gráficos & Plataformas Suportadas \\
\hline Game Maker $^{2}$ & 2D & Windows, Android, IOS, Ubuntu e Mac OS X \\
\hline${\text { Construct } 2^{3}}^{3}$ & 2D & $\begin{array}{l}\text { Windows, Windows Ohone, Android, IOS } e \\
\text { BlackBerry }\end{array}$ \\
\hline Unity $^{4}$ & 2D e 3D & $\begin{array}{l}\text { Windows, Windows Phone, BlackBerry 10, PS2, } \\
\text { PS3 e Web }\end{array}$ \\
\hline$U D K^{5}$ & 2D e 3D & Windows, Android, IOS, Mac OS X, PS2, PS3, PS4 \\
\hline
\end{tabular}

Em função das observações relatadas pelos professores de Matemática e pelos profissionais de Psicologia nas fases anteriores do trabalho, bem como pela necessidade de um desenvolvimento do jogo em gráficos $2 D$ para plataforma Windows e futura

\footnotetext{
${ }^{2}$ Para mais informações, consultar: www.yoyogames.com

${ }^{3}$ Para maiores informações, consultar: www.scirra.com

${ }^{4}$ Para maiores informações, consultar: www.unity3d.com

${ }^{5}$ Para maiores informações, consultar: www.unrealengine.com/udk/
} 
exportação para plataformas móveis, fazendo preferencialmente uso de programação drag-and-drop baseada em eventos, como sugerido por estes profissionais, optou-se pela ferramenta Construct 2, que dispõe ainda de uma documentação extensa e gratuita. Complementarmente, usou-se ainda o Corel Draw ${ }^{6}$, programa de desenho vetorial bidimensional para design gráfico, que permitiu criar as imagens usadas no jogo.

Depois de escolhida a ferramenta a ser usada no desenvolvimento do jogo, deuse início ao desenvolvimento do mesmo. Inicialmente foram criadas no software Corel Draw as imagens usadas no jogo (tabuleiro, dado, telas e botões em geral) que na sequência foram adicionadas ao software Construct 2 e organizadas segundo os critérios estabelecidos nas fases anteriores, de modo a iniciar-se o trabalho de programação do jogo. O jogo foi dividido em 4 (quatro) telas, descritas a seguir:

- Início: Nesta tela os jogadores têm sua primeira interação com o jogo, assim como podem escolher o percurso inicial que irão percorrer no jogo.

- Créditos: Esta tela apresenta as informações sobre os desenvolvedores da versão digital do jogo.

- Regras: Nesta tela são apresentadas as regras do jogo, bem como exemplos que devem auxiliar os jogadores na execução do mesmo.

- Jogo: Nesta tela é apresentado o jogo. Esta assume fundamental importância para o mesmo, pois é nela que se encontra o objeto principal do software desenvolvido, o jogo em si.

Após a finalização da primeira versão digital do jogo (Imagem 2), esta foi submetida à avaliação por estudantes e professores, caracterizando uma avaliação focada no cliente, e ao mesmo tempo focada em especialistas, a avaliação, é ainda focada tanto nos aspectos computacionais de interface do jogo, quanto nos aspectos pedagógicos e psicopedagógicos do mesmo, sendo esta avaliação, feita em aspectos quantitativos e aspectos qualitativos.

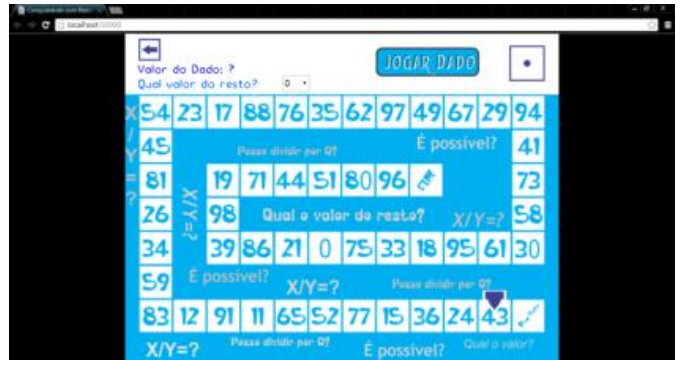

Imagem 2: Conquistando com Resto (versão digital)

\section{Avaliação do Jogo Conquistando com Resto}

A avaliação compreendeu tanto os aspectos de usabilidade quanto os aspectos pedagógicos do jogo. Neste sentido, foram escolhidos de forma aleatória, para avaliar o jogo, dez (de um total de quarenta) estudantes com idades entre dez e doze anos, integrantes do $6^{\circ}$ ano de uma escola pública estadual situada no Agreste do estado de Pernambuco. Além destes, colaboraram nesta etapa do trabalho: um professor de Matemática do ensino básico, um professor do ensino superior doutor em Matemática e

\footnotetext{
${ }^{6}$ Para maiores informações, consultar: www.corel.com
} 
uma profissional da área de Psicologia, os quais estiveram responsáveis por analisar as ações dos estudantes durante o processo de jogo.

\subsection{Avaliação de Interface}

A avaliação da interface foi realizada através de um questionário com o total de oito questões assertivas respondidas pelos estudantes. As questões envolveram itens de interface relacionados à aprendizagem, memorização e conforto visual, adaptados de Nielsen (1994). Optou-se por realizar esta avaliação de forma quantitativa, fazendo uso da escala de Likert, que em conformidade com Santos et al. (2014) permite mostrar qual o grau de concordância/discordância do usuário em relação aos elementos funcionais de determinado objeto.

Foram apresentadas aos estudantes as seguintes assertivas: (1) O jogo reconhece meus toques no mouse e teclado (2) As cores do jogo são agradáveis (3) Entendi o que cada ícone representa (4) A interface possui informações de fácil entendimento (5) Entendi rapidamente o que deveria fazer no jogo (6) É mais fácil aprender matemática com o jogo Conquistando com o Resto (7) O jogo é motivador e me deixa com vontade de continuar (8) Instalaria o jogo no meu computador ou dispositivo móvel e recomendaria a um amigo.

Para cada uma das assertivas apresentadas, os estudantes puderam escolher uma entre cinco diferentes alternativas: Concordo Fortemente (CF); Concordo (C); Indeciso (I); Discordo (D); Discordo Fortemente (DF). No Quadro 2 são apresentados os resultados colhidos durante a avaliação.

\section{Quadro 2: Resultados Quantitativos da Interface}

\begin{tabular}{|c|c|c|c|c|c|}
\hline \multicolumn{7}{|c|}{ Resultados Quantitativos da Interface } \\
\hline Assertiva/ Opção & CF & C & I & D & DF \\
\hline 1 & $20 \%$ & $60 \%$ & $10 \%$ & $10 \%$ & $0 \%$ \\
\hline 2 & $10 \%$ & $60 \%$ & $30 \%$ & $0 \%$ & $0 \%$ \\
\hline 3 & $40 \%$ & $20 \%$ & $30 \%$ & $10 \%$ & $0 \%$ \\
\hline 4 & $20 \%$ & $40 \%$ & $40 \%$ & $0 \%$ & $0 \%$ \\
\hline 5 & $20 \%$ & $20 \%$ & $50 \%$ & $10 \%$ & $0 \%$ \\
\hline 6 & $50 \%$ & $50 \%$ & $0 \%$ & $0 \%$ & $0 \%$ \\
\hline 7 & $50 \%$ & $40 \%$ & $10 \%$ & $0 \%$ & $0 \%$ \\
\hline 8 & $70 \%$ & $30 \%$ & $0 \%$ & $0 \%$ & $0 \%$ \\
\hline
\end{tabular}

Os resultados obtidos na etapa de avaliação da interface oportunizaram perceber que o jogo reconhece os movimentos e toques realizados pelos jogadores, tornando fácil a realização de jogadas. Foi possível perceber também a aceitação dos estudantes em relação às cores do jogo, tornando agradável o ato de jogar e, por conseguinte corroborando para que o mesmo possa permanecer por mais tempo no jogo.

Observou-se ainda, que todos os estudantes concordaram que é mais fácil compreender o conceito de divisão com o uso do jogo, bem como acreditam que o mesmo é motivador e os instigam a permanecer por mais tempo no jogo. Todos concordaram ainda que também instalariam o jogo em seu dispositivo móvel ou em seu computador, demostrando que os mesmos se mostraram satisfeitos com o jogo, 
motivados para continuar a jogar e dispostos a continuar a aprender os conceitos apresentados no jogo.

\subsection{Avaliação Pedagógica}

$\mathrm{Na}$ avaliação dos aspectos pedagógicos do jogo, optou-se por uma avaliação qualitativa, de modo que foi entregue a cada estudante uma folha de rascunho a fim de que pudessem usá-la para ajudá-los a realizar os cálculos necessários para a realização das jogadas, estas folhas foram posteriormente analisadas pelos professores.

Nesta etapa da avaliação observou-se que nove dos dez estudantes utilizaram a folha de rascunho para realizar alguns dos cálculos necessários para a conclusão das jogadas (apenas um estudante realizou todos os cálculos mentalmente, sem fazer uso da folhe de rascunho), denotando que os estudantes não abandonaram os mecanismos didáticos tradicionais ao mesmo tempo em que fizeram uso de um mecanismo digital contemporâneo.

Outro aspecto relevante observado foi à dificuldade enfrentada pela maioria dos estudantes ao se deparar com um cálculo cujo quociente fosse um número decimal, os estudantes apresentavam dificuldade em usar o elemento "resto" e acabavam por utilizar sucessivas tentativas de multiplicação para alcançar a resolução da questão.

Pode-se perceber ainda que a maioria dos discentes notou que seria difícil encontrar um número no dado que apresentasse um divisor não exato para os números 24 e 36, de modo que alguns questionaram este fato. Tal acontecimento permite ao docente esclarecer uma série de conceitos matemáticos quando questionado em sala de aula.

Da mesma forma aconteceu em relação ao número zero, em que os estudantes testaram todas as possibilidades de divisão com relação a este número e puderam observar que é impossível a divisão por zero, o que os levou a questionarem este fato. Através destes resultados foi possível concluir que o jogo conseguir abordar uma operação aritmética da Matemática, cumprindo assim com seus objetivos pedagógicos abordados em sua versão tradicional.

\section{Considerações Finais}

De acordo com as pesquisas de Santos et al. (2013), que apontam a disparidade entre os aspectos pedagógicos e psicopedagógicos dos jogos tradicionais em comparação com os jogos digitais usados no ensino da Matemática, acredita-se que a virtualização de jogos, ora apresentada, oferece uma alternativa para auxiliar o processo de ensino e aprendizagem, atrelando os conceitos e paradigmas pedagógicos e psicopedagógicos da versão tradicional de um jogo a elementos contemporâneos dos jogos digitais, promovendo assim uma união entre o tradicional e o digital.

Ainda neste sentido, o jogo então apresentado, torna-se uma possibilidade para que professores e estudantes possam usufruir de um ambiente lúdico e interativo, mantendo-se os aspectos pedagógicos e psicopedagógicos, presentes na versão tradicional do jogo e ao mesmo tempo, atrelado às novas mecânicas de jogos digitais.

Os resultados obtidos oportunizaram perceber que o jogo foi aceito de forma positiva por estudantes e professores, percebeu-se também que o jogo foi avaliado de forma positiva tanto com relação a sua usabilidade, quanto aos seus aspectos 


\section{CBIE-LACLO 2015}

Anais dos Workshops do IV Congresso Brasileiro de Informática na Educação (CBIE 2015)

pedagógicos matemáticos. Assim, os resultados deste trabalho corroboraram não somente com o desenvolvimento do jogo enquanto objeto de ensino, mas também com relação ao processo de virtualização e o processo de avaliação do jogo, que pode ser replicado para outros projetos de cunho similar.

Espera-se com os resultados obtidos, promover a Virtualização de Jogos como um recurso capaz de unir os aspectos tradicionais aos aspectos digitais em favor da educação, estimulando o uso de jogos na educação, especialmente na disciplina de Matemática, alvo desta pesquisa. Como trabalhos futuros, desafia-se ainda vivenciar novos testes em diferentes ambientes e maior quantidade de estudantes para este jogo, bem como submeter outros jogos tradicionais ao processo de virtualização (atividade já em andamento), ${ }^{7}$ além de promover novos tipos de aplicação e validação para os mesmos, bem como fomentar a Virtualização de Jogos em nível de pesquisa acadêmica.

\section{Referências}

Araújo, B. M. R., Freitas, C. M. S. M., Caminha, I. O., Silva, P. P. C. Virtualização esportiva e os novos paradigmas para o movimento humano. Revista Motriz. Vol. 17, n. 4, 2011.

Buchinger, D., Hounsell, M.S., Dias, C (2012) "Colaboratividade em um Jogo Eletrônico para Ensino sobre Dengue", In: anais do XXIII Simpósio Brasileiro de Informática na Educação (SBIE), Rio de Janeiro-RJ.

Cabreira, L. G., Aquino, O., R. (2006) "Jogos eletrônicos - a virtualização do brincar na perspectiva dos professores de $3^{\mathrm{a}}$ e $4^{\mathrm{a}}$ séries do ensino fundamental I de uma escola particular de Maringá/PR”, In: Educação em Revista 7 ed. p. 85-102, São Paulo-SP.

Gladcheff, A., P.; Zuffi, E., M.; Silva, M., D. (2011) "Um Instrumento para Avaliação da Qualidade de Softwares Educacionais de Matemática para o Ensino Fundamental". In: Anais do XVII Workshop de Informática na Escola (WIE), Fortaleza-CE.

Medeiros, T. J., da Silva, T. R., Aranha, E., H., S. (2013) "Ensino de programação utilizando jogos digitais: uma revisão sistemática da literatura". In: Revista Novas Tecnologias na Educação (RENOTE), v.11, n.2, Porto Alegre - RS.

Nielsen, J. (1994). Usability engineering. Elsevier.

Reinoso, L., F., Neto, C., B., Lopes, L., C., L. (2012) "Principais Características dos Games para serem Inseridos como Ferramenta Educacional", Revista Novas Tecnologias na Educação (RENOTE), v.11, n.2, p. 42-43, Porto Alegre - RS.

Ribeiro, A., L. (2012) "Jogos digitais online: ampliando o(s) Letramento(s) na sala de aula" In: Anais do V Simpósio Hipertexto e Tecnologias na Educação (SHTE), Recife PE, Brasil.

\footnotetext{
${ }^{7}$ Outros jogos já vêm sendo submetidos por este grupo de pesquisadores ao processo de Virtualização de Jogos, bem como a diferentes tipos de avaliação, como é o caso do jogo "Desafio com Palitos" que tem seu processo de desenvolvimento descrito no artigo "Desafios com Palitos: Processo de Desenvolvimento de um Jogo Educativo" publicado nos anais do XIII Simpósio Brasileiro de Jogos e Entretenimento Digital (SBGames 2014) e disponibilizado neste link: http://migre.me/oxlqf, entre outros.
} 


\section{CBIE-LACLO 2015}

Anais dos Workshops do IV Congresso Brasileiro de Informática na Educação (CBIE 2015)

Sá, E., J., V., Teixeira, J., S., F., Fernandes, C., T. (2007) "Design de atividades de aprendizagem que usam Jogos como princípio para Cooperação", In: Anais do XVIII Simpósio Brasileiro de Informática na Educação (SBIE), São Paulo - SP, Brasil.

Santos, W. O., da Silva, A. P., Silva Junior, C. G. (2014) "Conquistando com o Resto: Virtualização de um Jogo para o Ensino de Matemática”. In: Anais do XXV Simpósio Brasileiro de Informática na Educação (SBIE). p. 317-321. Dourados MT.

Santos, W. O., Silva Junior, C. G., Barros, F. L. P. (2013) "Processo de virtualização de jogos matemáticos tradicionais, para uso como ferramenta de auxilio ao processo de ensino e aprendizagem em escolas do agreste de Pernambuco", In: Anais do III Encontro Anual de Tecnologia da Informação (EATI), Frederico Westphalen - RS, p. 312-316.

Santos, W. O., Silva Junior, C. G. (2014). "Uso de Jogos no ensino da Matemática: Uma análise entre os jogos tradicionais e os jogos digitais, baseada em pesquisa e mapeamento dos materiais encontrados na Web". In: Anais do X Seminário Jogos Eletrônicos Educação e Comunicação (SJEEC). Salvador - BA.

Santos, W. O., Silva Neto, S. R., Silva Junior, C. G. (2013) "Uso de Games no ensino da Matemática. Uma proposta de virtualização dos jogos tradicionais, para uso como mecanismo de apoio ao processo de ensino e aprendizagem", In: Anais do V Simpósio Hipertexto e Tecnologias na Educação (SHTE), Recife - PE, p. 216.

Santos, W. O., Silva Neto, S. R., Silva Junior, C. G. (2015). "Processo de Virtualização de Jogos para Uso como Mecanismo de Apoio ao Processo de Ensino e Aprendizagem da Disciplina de Matemática”. In: Anais do XI Seminário Jogos Eletrônicos Educação e Comunicação (SJEEC). Salvador - BA, p. 109-117.

Santos, W. O., Silva Neto, S. R., I., I., Bittencourt, Silva Junior, C. G. (2015) "Avaliação de Jogos Educativos: Uma Abordagem no Ensino de Matemática". In: Anais do XXVI Simpósio Brasileiro de Informática na Educação (SBIE). Maceió - AL.

Silva, E. G., Barbosa, A. F., Silva Neto, S., R., Santos, W. O., Rodrigues, A., N. (2014) "O processo de desenvolvimento do jogo" Mundo de Euclides" para ensino da Geometria Euclidiana”. In: Anais do XIII Simpósio Brasileiro de Jogos e Entretenimento Digital (SBGames) p. 942-945. Porto Alegre - RS.

Silva Neto, S. R., Santos, H. R. M., de Souza, A. A., \& Santos, W. O. (2013). “Jogos Educacionais como Ferramenta de Auxílio em Sala de Aula". In Anais do XIX Workshop de Informática na Escola (WIE). Campinas-SP, p. 130-139.

Togni, A. C., Rehfeldt, M. J. H., Bersch, M. E., Thomas, M. I. P., Poletti, C. F., \& Kronbauer, K. A. (2009). Piff geométrico: um objeto virtual de aprendizagem para o ensino de ciências exatas. In: Revista Novas Tecnologias na Educação (RENOTE), v.7 n.3, 45-52.

Zorzal, E. R., Kirner, C., Cardoso, A., Lamounier JR, E., de Oliveira, M. R. F., \& Silva, L. F. (2008). Ambientes Educacionais Colaborativos com Realidade. Aumentada. In: Revista Novas Tecnologias na Educação (RENOTE), v.6 n.1. 